\title{
Longitudinal methods for life course research: A comparison of sequence analysis, latent class growth models, and multi-state event history models for studying partnership transitions
}

\author{
Júlia Mikolai University of St Andrews and University of Southampton, UK \\ Julia.Mikolai@st-andrews.ac.uk
}

Mark Lyons-Amos London School of Hygiene and Tropical Medicine and University of Southampton, UK

(Received May 2016 Revised January 2017)

http://dx.doi.org/10.14301/Ilcs.v8i2.415

\begin{abstract}
This paper qualitatively compares and contrasts three methods that are useful for life course researchers; the more widely used sequence analysis, and the promising but less often applied latent class growth models, and multi-state event history models. The strengths and weaknesses of each method are highlighted by applying them to the same empirical problem. Using data from the Norwegian Generations and Gender Survey, changes in the partnership status of women born between 1955 and 1964 are modelled, with education as the primary covariate of interest. We show that latent class growth models and multi-state event history models are a useful addition to life course researchers' methodological toolkit and that these methods can address certain research questions better than the more commonly applied sequence analysis or simple event history analysis.
\end{abstract}

\section{Keywords}

Life course methodology, sequence analysis, latent class growth models, multi-state event history models, Norway, partnership transitions, family life course

\section{Introduction}

In the last half century, family life courses have changed considerably. For example, the transition to parenthood has been delayed, non-marital cohabitation and non-marital childbearing have become more common, as have union dissolution and re-partnering. Additionally, the timing and sequencing of family life events has changed resulting in more complex and less predictable family life courses. These changes have generated an increased interest in the applicability of different methods for modelling life courses with their complexities. Although a number of methods are available to study the family life course, discussion has mainly been limited to comparing simple event

history models and sequence analysis (Billari, 2001b, 2005; Billari \& Piccarreta, 2001, 2005; Piccarreta \& Billari, 2007).

Simple event history analysis is commonly used to examine single or competing events (Heuveline \& Timberlake, 2004; Perelli-Harris \& Gerber, 2011; Perelli-Harris, Sigle-Rushton, et al., 2010). Applications of event history analyses vary in focus and complexity. For example, recent studies (Baizán, Aassve, \& Billari, 2003, 2004) applied simultaneous equations models to study the determinants of several concurrent life course transitions. Others used multilevel multiprocess models to account for correlated event histories (Steele, Kallis, Goldstein, \& Joshi, 2005). These 'event based' approaches 
primarily focus on the (causal) influence of certain covariates on particular events. Simultaneous models improve upon simple event history models by accommodating possible interdependencies between several events via modelling joint processes and unobserved heterogeneity. Even so, they limit attention to studying a specific segment of the life course.

Others have promoted the use of sequence analysis arguing that, unlike event history analysis, this 'holistic approach' examines the life course trajectory as a whole meaningful unit. This technique creates 'ideal-types' of trajectories that categorise and describe different life course patterns (Billari, 2001a, 2001b, 2005; Billari \& Piccarreta, 2005; Piccarreta \& Billari, 2007). It is then possible to assess how different covariates influence the probability of an individual to belong to one of these 'ideal-types'.

Despite the availability of other techniques only a few studies have investigated their applicability to life course research. For example, Barban and Billari (2012) have compared and tested the consistency of sequence analysis and latent class analysis. Additionally, Bonetti, Piccarreta, and Salford (2013) proposed an extension of multi-state models to studying the family life course. This paper aims to contribute to this line of research by qualitatively comparing the strengths and weaknesses of sequence analysis and two other promising techniques: latent class growth models, and multistate event history models. These methods combine the properties of the event based and the holistic approaches by focusing on several consecutive events and thus are ideal to examine the family life course.

By applying these methods to a real life example, the differences and similarities as well as the strengths and weaknesses of these approaches are emphasised. Our application focuses on the role of education on changes in partnership status (i.e. being never partnered, transition to first cohabitation and first marriage, the dissolution of a first cohabitation or a first marriage, and forming a new partnership after union dissolution) of Norwegian women born between 1955 and 1964 . We ask the following questions, pertinent to life course research: how can sequence analysis, latent class growth models and multi-state event history models be used for studying the influence of education on partnership transitions over the early family life course? What types of research questions can be answered using these methods? And are these methods applicable to the same problems to the same extent or is one of them better than the other and if so in which situation?

\section{Data}

We illustrate similarities and differences between sequence analysis, latent class growth models, and multi-state event history models using data from the first wave of the Norwegian Generations and Gender Survey ${ }^{i}$ (GGS) from $2007 / 2008$. We examine the influence of educational attainment on changes in partnership status of women born between 1955 and 1964 ( N = 1290). The dataset includes extensive retrospective information on the start and end date (year and month) of up to five cohabitating and marital unions as well as union dissolutions. Cohabitation is defined as a co-residential relationship which lasted for at least three months. For this application Norwegian data are used because in Norway variation in partnership experiences is one of the largest compared to other European countries (Elzinga \& Liefbroer, 2007). This enables us to demonstrate the strengths and weaknesses of the examined methods using a rich dataset with more complex partnership experiences.

Although the Norwegian GGS provides crosssectional weights, not all applied methods are able to incorporate these. Therefore, the analyses presented in this paper do not incorporate weights. This implies that the results might not be representative of the overall distribution of the examined partnership formation behaviours in Norway. However, this is not a major limitation because the aim is not to provide population estimates but to explore how the different methods can be applied to the same problem.

\section{Methods and Modelling Strategy}

\section{Sequence Analysis}

Sequence analysis (SA) represents each individual life course by a sequence (i.e. a character string, which indicates the order and duration of states occupied by an individual in each month). For example, the sequence SSSCCMMMM means that the respondent was never partnered ${ }^{\text {ii }}(\mathrm{S})$ for three months followed by two months of cohabitation (C) and four months of marriage (M). Due to the large possible number of combinations of states, usually 
very few individuals experience the exact same sequence. To reduce the number of sequences, Optimal Matching Analysis (OMA) is used.

OMA is a technique that measures the dissimilarity between sequences by identifying how similar pairs of sequences are. Similarity is defined in terms of the number, order, and duration of states within sequences. The algorithm calculates the similarity/dissimilarity between two sequences by taking into account three possible operations: replacement (one state is replaced by another one), insertion (an additional state is added to the sequence), and deletion (a state is deleted from the sequence). The fewer operations are needed to turn one sequence into the other, the more similar two sequences are and vice versa. Furthermore, to each operation, a certain cost can be attached. Therefore, identifying the relative cost of all operations is critical to determine the (dis)similarity between sequences. These require a priori definition by the researcher with little objective measure of the correct specification, and the results can be highly sensitive to these specifications (Brzinsky-Fay \& Kohler, 2010). Then, the distance between two sequences is defined by the minimum cost of the operations that are necessary to transform one sequence into the other (Abbott \& Tsay, 2000). The distances are recorded in a dissimilarity matrix.

Then, in order to find existing patterns in the data, hierarchical cluster analysis is performed on the dissimilarity matrix. The aim of the cluster analysis is to minimise the within-cluster and maximise the between-cluster distance. The researcher needs to specify the number of clusters to be extracted from the data either a priori or by using fit statistics. Once the clusters are formed, they can be described with respect to the variables used to create the clusters (in this example partnership experiences). The clusters can be used both as independent and dependent variables in further analyses. Additionally, sequences can also be compared based on the number of episode changes within once sequence, the length of the sequences, or the number of different events in a sequence (Brzinsky-Fay \& Kohler, 2010).

In our application, clusters are created based on women's monthly partnership trajectories between age 15 and 40 . Women can be in the following partnership states in a given month: single, cohabiting, married, and separated. Individuals who experience a new partnership following separation can be in the 'marriage' or 'cohabitation' state. After performing OMA with equal costs assigned to indel operations (i.e. insertion and deletion; in this instance 1), individuals are allocated to clusters based on Ward's distance. Since the results can be sensitive to the chosen indel and substitution costs, we performed sensitivity analyses varying the indel costs to be $0.5,1.0$ and 1.5, and using both a constant substitution matrix as well as a matrix based on the frequency of transitions. The findings indicated that the number and composition of the obtained clusters remain consistent across the different specifications. We assess the number of clusters based on two measures of average cluster linkage; the Calinski-Harabasz pseudo- $F$ index (Calinski \& Harabasz, 1974) and the Duda-Hart index (Duda \& Hart, 1973). These statistics help to determine the optimal number of clusters by comparing the ratio of the within-cluster distances to the between-cluster distances. Additionally, the Duda-Hart index also produces a pseudo T-statisticiii. Once the optimal number of clusters is established, cluster allocation is used as the response variable in a multinomial logistic regression. The models are estimated using the SQ-Ados for Stata 12 (BrzinskyFay, Kohler, \& Luniak, 2006).

\section{Latent Class Growth Models}

Latent Class Growth Models (LCGMs) are an extension of conventional growth curve models with the assumption that individuals are drawn from different subpopulations (latent classes) that have different growth trajectories (Perelli-Harris \& Lyons-Amos, 2015). Similarly to SA, these models have an individual centred perspective, thus they seek to identify relationships between individual response patterns and form groups based on these patterns (Jung \& Wickrama, 2008). Individuals' relationship histories are recorded at each age and then grouped into latent classes. The response (in this application partnership state in each year) is defined as the random variable $y_{i, a g e}$ with the following categories: never partnered $(0)$, persistent cohabitation (1), marriage (2), and separated (3). After separation, individuals are allowed to re-enter cohabitation or marriage. Respondents move between these partnership states between age 15 and $40^{\mathrm{iv}}$.

The specification of the growth curves and the robustness checks are similar to those in PerelliHarris and Lyons-Amos (2015). Classes are formed 
based on yearly partnership histories ${ }^{\mathrm{V}}$. Individuals' partnership histories form trajectories which are combined to form the latent classes. Latent classes describe different partnership patterns across individuals' life courses. Each woman has a probability of belonging to each latent class. The closer an individuals' partnership history is to the class trajectories, the more likely she is to belong to a particular latent class. The probability of being in partnership state $s$ at a given age is defined as $\pi_{i, a g e}^{s}=P\left(y_{i, a g e}=s\right)$ where $i$ stands for individuals. The probability of each partnership (compared to marriage which is the reference category) across the life course is modelled as a growth equation (see Equation 1). A separate growth equation is specified for each class $C_{j}(j=$ $1 \ldots 5)$ which are defined by a class-specific intercept $\left(\alpha_{j}^{S}\right)$ and class-specific slope parameters $\left(\beta_{1 \ldots 4, j}^{S}\right)$.

$\ln \left(\frac{\left(\pi_{i, a g e}^{S} \mid C_{j}=j\right)}{\left(\pi_{i, a g e}^{S=2} \mid C_{j}=j\right)}\right)=\alpha_{j}^{s}+\beta_{1, j}^{s} a g e_{i}+$
$\beta_{2, j}^{s} a g e_{i}^{2}+\beta_{3, j}^{s}$ educ $+\beta_{4, j}^{s}$ educ $* a g e_{i}$

In order to examine the relationship between the latent classes and education LCGMs offer two possibilities. First, education can be used to predict the probability of belonging to a certain latent class (Wang, Hendricks Brown, \& Bandeen-Roche, 2005). This approach is comparable to SA. Where LCGMs have an advantage over SA is that they can also allow for education to alter the shape of the growth curves. The parameter $\beta_{3, j}^{S}$ expresses how the inclusion of educational level alters the intercept while $\beta_{4, j}^{S}$ indicates the influence of education on the slope of the growth curves. An additional advantage of LCGMs compared to SA is that a variety of fit statistics are available for deciding the optimal number of classes and this choice can be validated via simulation since the estimates are model based. However, the different criteria and test statistics (such as AIC, BIC or Lo-Mendell-Rubin Likelihood Ratio Test) can lead to different and sometimes contradictory conclusions (Nylund, Asparouhav, \& Muthen, 2007).

In our application, we explore a set of two, three, four, and five class models and perform the LoMendell-Rubin-Likelihood Ratio Test (LMR-LRT) for all classes. This test examines the improvement in model fit for a J class model compared to a J-1 class model. In case of a two class model, it is equivalent to testing whether the LCGM performs better than a simple latent growth curve model, which assumes that one growth curve is enough to describe women's partnership behaviours. The models are estimated in Mplus 6.2 for Linux. Note that we do not explore models with more than five classes ${ }^{\mathrm{vi}}$. Due to the specification of partnership state as a nominal variable, the implementation of this model is not part of the main Mplus language. As a result, model estimation is computationally intense both due to the difficulty of the calculations required and the volume of data to be read.

\section{Multi-state Event History Models}

Multi-state event history models differ from SA and LCGMs in that they do not aim to classify or group individuals ${ }^{\text {vii. }}$. It is a variable-centred approach where the main purpose is to establish statistical relationships between the independent variable(s) and several transitions. Multi-state event history models are an extension of simple event history models; rather than examining one transition, this approach allows individuals to move among different states over time. These movements are assumed to be stochastic and are modelled by means of transition probabilities. Thus, multi-state event history models allow for examining covariate effects on several transitions within the same model. 


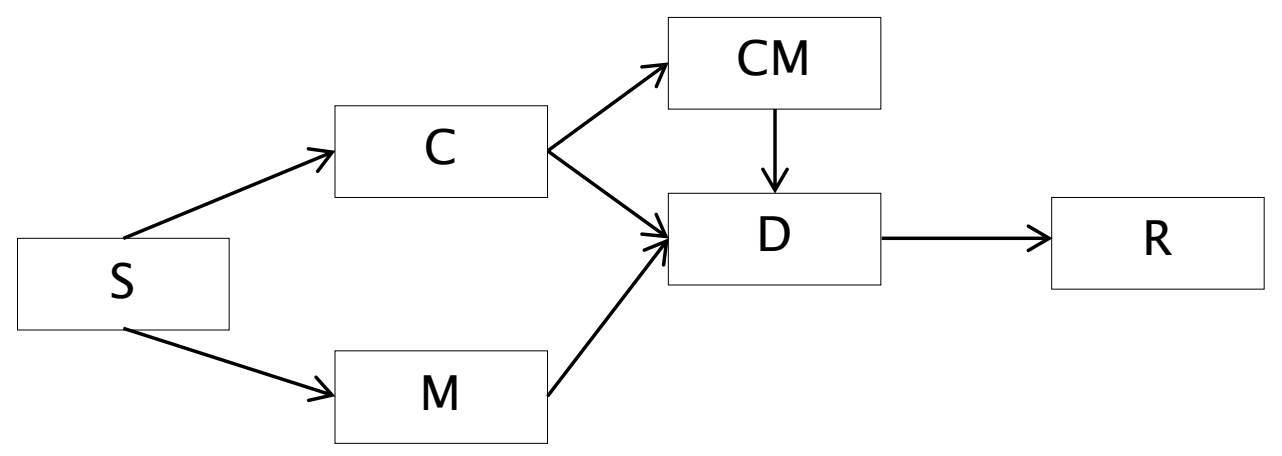

Note: $S$ - never partnered, $C$ - cohabitation, $M$-direct marriage, CM-marriage preceded by cohabitation with the same partner, $D$ - union dissolution, $R$-re-partnering.

\section{Figure 1. Multi-state event history model}

Another distinct advantage of this method is the possibility to include time-varying covariates and thereby examine how the influence of a variable of interest changes over the family life course. For example, it is possible to examine the influence of educational attainment, which may change over the life course, on several family life transitions. This cannot be done using simple event history models, SA, or LCGMs. Multi-state models assume the Markov property; that is that the present behaviour of an individual is enough to predict their future behaviour (Andersen \& Keiding, 2002; Hougaard, 1999). For example, it would assume that the transition probability from marriage to union dissolution is the same for all individuals regardless of whether they have cohabited before marriage. As life course theory emphasises that earlier transitions play an important role in later transitions, this assumption is not realistic. In order to be able to examine the partnership transitions in a dynamic way, the model can be extended. We do so by defining the state ' $\mathrm{CM}$ ' to differentiate between direct marriage and marriage that was preceded by cohabitation. One disadvantage of multi-state event history models is that as the number of states increases and as individuals move along the life course, one might end up with small cell sizes and thus, with unreliable estimates of the transition hazards.

Figure 1 shows the multi-state event history model, where the following states are defined: never partnered (S), cohabitation (C), direct marriage $(\mathrm{M})$, marriage that was preceded by cohabitation with the same partner (CM), union dissolution (D) and re-partnering (R). We do not distinguish between cohabitation and marriage as a form of re-partnering due to relatively small cell sizes and to keep the models comparable. The multi-state event history model is estimated using a stratified continuous-time Cox model with a nonparametric baseline hazard where each transition is represented by a different stratum (de Wreede, Fiocco, \& Putter, 2011; Putter, Fiocco, \& Geskus, 2007; Putter, van der Hage, de Bock, Elgalta, \& van de Velde, 2006). Covariates are incorporated as transition-specific covariates to allow for their effect to differ across transitions. The transition hazard of individual $k$ is given by:

$$
\lambda_{i j}(t \mid \boldsymbol{Z}(k))=\lambda_{i j, 0}(t) \exp \left(\boldsymbol{\beta}_{\boldsymbol{i j}}{ }^{T} \boldsymbol{Z}(k)_{i j}\right)
$$

where $i j$ indicates a transition from state $i$ to state $j$, $\lambda_{i j, 0}(t)$ is the baseline hazard, $Z(k)$ is the vector of covariates at baseline for individual $k$ and $\boldsymbol{Z}(k)_{i j}$ is the vector of transition-specific covariates for individual $k$.

In this application, we use monthly information on partnership experiences. To estimate the model, an augmented dataset is used with one row per transition that the individual is at risk of. Women are observed from age 15, when they are never partnered until age 40, the time of the survey, or the time when they experience re-partnering, whichever happens earlier. As educational 
attainment is defined as a time-varying categorical variable, additional episode splitting is performed where an educational transition happens within an at-risk period. The models are estimated using the mstate package in $\mathrm{R}$ (de Wreede et al., 2011).

\section{Variables}

Level of education. In all three models, the highest level of education at the time of the survey is measured by a variable with the following categories: Iow (ISCED 0 to ISCED 2), medium (ISCED 3 and ISCED 4), and high education (ISCED 5 and ISECD 6). High education is the reference category in all three models. In the multi-state event history models, education is measured as a time-varying variable which is created using information on the year and month of reaching the highest level of education. We assume continuous education from age 15 and that secondary education takes four years while tertiary education takes three years on average. Missing information (7.9\%) on the year and/or month of reaching the highest level of education was imputed using information on the median age of finishing education by educational level. In LCGMs and SA, education is time-constant and indicates the highest level of education at the time of the survey.

\section{Results}

\section{Sequence Analysis}

Table 1 presents the Calinski-Harabasz and the Duda-Hart indices for two to seven cluster models. On the Calinski-Harabasz and Duda-Hart indices, higher values indicate more distinct clustering, whereas for the related Duda-Hart Pseudo T-square measure, lower values are indicative of more distinct grouping.

\section{Table 1. Calinski-Harabasz and Duda-Hart indices for $k$ cluster specifications}

\begin{tabular}{cccc}
$\begin{array}{c}\text { Number of clusters } \\
(\mathrm{k})\end{array}$ & $\begin{array}{c}\text { Calinski-Harabasz } \\
\text { Pseudo-F }\end{array}$ & \multicolumn{2}{c}{ Duda-Hart indices } \\
\hline 2 & 51.39 & 0.97 & Pseudo T-square \\
\hline 3 & 33.72 & 0.95 & 12.96 \\
4 & 35.73 & 0.52 & 42.33 \\
5 & 81.37 & 0.98 & 227.69 \\
6 & 67.79 & 0.99 & 8.91 \\
7 & 56.79 & 0.99 & 1.22 \\
\hline
\end{tabular}

Note: Numbers in boldface indicate the best fit for the given index.

There is disagreement between these indices as to the optimal number of clusters. The CalinskiHarabasz index indicates a five cluster solution while the Duda-Hart indices indicate a six cluster solution to be optimal. We proceed with a six cluster model because the sixth cluster has a substantial, distinct meaning for our application.

The six clusters are shown in six rows of figure 2 . The graphs in the left hand column show so called sequence index plots where each line represents the partnership sequences of an individual; these individual sequences are stacked on top of each other. The graphs in the right hand column are so called chronograms, which represent the distribution of states at each age providing a more readable summary than sequence index plots (Halpin, 2014).

The first cluster (figure 2, first row) is characterised by late partnership formation, where the first partnership is either direct marriage or cohabitation. Therefore, this cluster is titled 'late, varied partnerships'. Women who belong to the second cluster form partnerships at a much younger age, than those in the first cluster (figure 2, second row). Most of these partnerships start as direct marriage, and there is a large degree of union instability at later ages. This cluster is, thus, named the 'early, direct, unstable marriage' cluster. The third cluster (figure 2, third row) is largely characterised by stable cohabitation across the life course, although for some individuals cohabitation 
is a second partnership following the dissolution of a marriage. This cluster is named the 'cohabitation' cluster. Cluster four (figure 2, fourth row) consists of women whose first union is direct marriage starting from about the age of 25 . There is little evidence of any other partnership behaviour, and for this reason cluster four is called 'later, direct, and stable marriage' cluster. Cluster five (figure 2, fifth row) captures women who form cohabiting unions, which then transition to marriage. We, therefore, call this cluster 'cohabitation followed by marriage' cluster. Finally, the sixth cluster (figure 2, sixth row) comprises of women who married their partner directly at a (very) young age (before age 25). There is very limited evidence of any other partnership form (<5\%) in this cluster, hence we call this cluster the 'early, direct, and stable marriage' cluster.
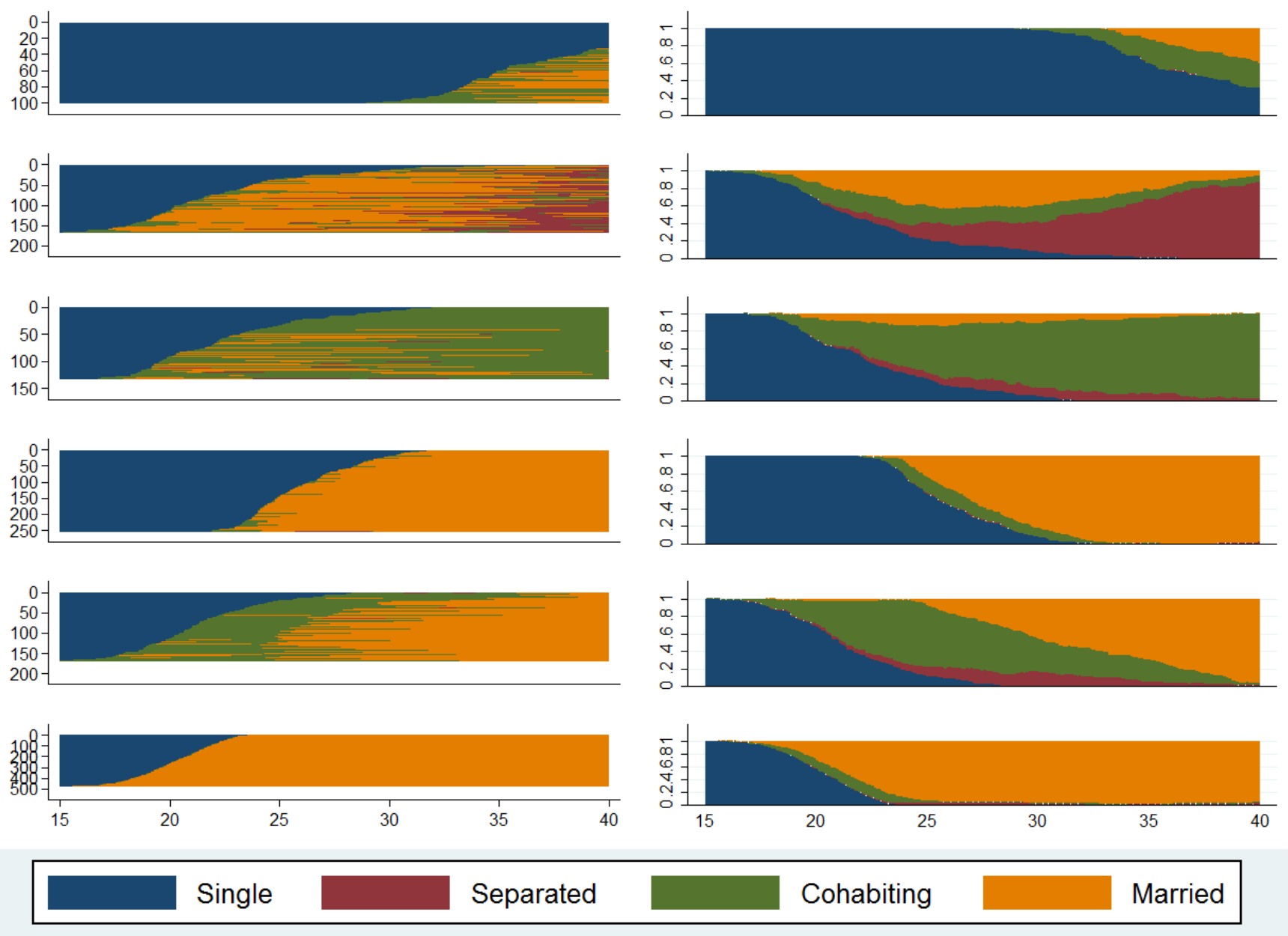

Note: Each row corresponds to a cluster.

Note: Cluster 1: Late, varied partnerships (7.8\%); Cluster 2: Early, direct, unstable marriage (12.8\%); Cluster 3: Cohabitation (10.2\%); Cluster 4: Later, direct, and stable marriage (19.5\%); Cluster 5: Cohabitation followed by marriage (13.0\%); Cluster 6: Early, direct, and stable marriage (36.8\%).

\section{Figure 2. Results of sequence analysis in the form of sequence index plots (left hand column) and chronograms (right hand column)}

After having identified these six clusters, we apply multinomial logistic regression to assess how educational attainment influences the likelihood of women to belong to one of the six clusters (table 2). We compare the likelihood of belonging to each cluster to the likelihood of belonging to cluster six 
(early, direct, and stable marriage) because this cluster is the largest. To further facilitate the interpretation of the regression coefficients, predicted probabilities are calculated (table 3). The results show that lower educated women are significantly less likely to belong to the late, direct, and stable marriage' cluster (cluster four) and to the 'cohabitation followed by marriage' cluster (cluster five) compared to belonging to the 'early, direct, and stable marriage' cluster than highly educated women.

Table 2. Results of the multinomial logistic regression, regression coefficients

\begin{tabular}{lccccc} 
& $\begin{array}{c}\text { Membership } \\
\text { of cluster 1 vs } \\
\text { cluster 6 }\end{array}$ & $\begin{array}{c}\text { Membership } \\
\text { of cluster 2 vs } \\
\text { cluster 6 }\end{array}$ & $\begin{array}{c}\text { Membership } \\
\text { of cluster 3 vs } \\
\text { cluster 6 }\end{array}$ & $\begin{array}{c}\text { Membership } \\
\text { of cluster 4 } \\
\text { vs cluster 6 }\end{array}$ & $\begin{array}{c}\text { Membership } \\
\text { of cluster 5 } \\
\text { vs cluster 6 }\end{array}$ \\
\hline $\begin{array}{l}\text { Education } \\
\text { High (ref) }\end{array}$ & & & & & \\
Medium & 0.088 & -0.151 & -0.107 & $-0.630 * * *$ & -0.169 \\
Low & -0.162 & -0.343 & -0.017 & $-1.220 * * *$ & $-0.405^{*}$ \\
& & & & & \\
Intercept & $-1.550^{* * *}$ & $-0.913^{* * *}$ & $-1.230^{* * *}$ & -0.151 & $-0.880^{* * *}$ \\
\hline Note: ${ }^{*} p<.05^{* *} p<.01{ }^{* * *} p<.001$ & & & &
\end{tabular}

Table 3. Predicted probabilities of cluster membership by educational level

\begin{tabular}{lccc} 
Cluster & Low education & Medium education & High education \\
\hline $\begin{array}{l}\text { 1 Late, varied partnerships } \\
\text { 2 Early, direct, unstable }\end{array}$ & 0.08 & 0.21 & 0.07 \\
$\begin{array}{l}\text { marriage } \\
\text { 3 Cohabitation }\end{array}$ & 0.12 & 0.16 & 0.13 \\
$\begin{array}{l}\text { 4 Late, direct, stable } \\
\text { marriage }\end{array}$ & 0.13 & 0.17 & 0.09 \\
$\begin{array}{l}\text { 5 Cohabitation followed by } \\
\text { marriage }\end{array}$ & 0.11 & 0.10 & 0.27 \\
$\begin{array}{l}\text { 6 Early, direct, stable } \\
\text { marriage }\end{array}$ & 0.12 & 0.16 & 0.13 \\
\hline
\end{tabular}

\section{Latent Class Growth Models}

Table 4 presents fit statistics for two-, three-, four-, and five class models. The LMR-LRT p-value indicates that the two-class model is an improvement over a one-class model, justifying the LCGM approach. All fit statistics indicate improving model fit with the addition of higher order classes.
From the examined models, the five-class model demonstrated the best model fit based on AIC, BIC and Sample Size BIC (SSBIC) statistics. Although the Lo-Mendell-Rubin Likelihood Ratio Test (LMR-LRT) indicates that a four-class model is adequate, we select a five-class model since this is the optimal number of classes according to all other fit statistics. 
Table 4. Fit statistics for two-, three-, four-, and five-class models

\begin{tabular}{ccccc} 
Number of classes $(\mathrm{J})$ & AIC & BIC & SSBIC & LMR-LRT (p-value) \\
\hline 2 & 138352.929 & 138731.851 & 138588.841 & 0.000 \\
3 & 132500.352 & 133081.366 & 132862.085 & 0.016 \\
4 & 129273.584 & 130056.690 & 129761.137 & $\mathbf{0 . 0 2 1}$ \\
5 & $\mathbf{1 2 6 7 2 5 . 4 9 9}$ & $\mathbf{1 2 7 7 1 0 . 6 9 7}$ & $\mathbf{1 2 7 3 3 8 . 8 7 1}$ & 0.174 \\
\hline
\end{tabular}

Note: Numbers in boldface indicate the best fit based on the given statistic.

Figure 3 presents partnership profiles for the five extracted classes by educational attainment. The latent classes are depicted in the rows and educational groups are shown in the columns (e.g. the graph in the first row and first column shows class one for low educated women). Class one captures early and varied partnership forms, with an initial increase in the probability of both cohabitation and marriage for all educational levels. The probability of marriage peaks around age 24 for high and medium educated and at age 22 for low educated, and declines thereafter. The probability of cohabitation rises, plateauing at age 22 for high and medium educated and at age 19 for low educated, before increasing again from around age 31 onwards. These relationships, formed at relatively early ages, are unstable; the probability of separation is high across all partnership forms and educational levels. There is some variation in how the probability of separation changes over age by educational attainment. For women with high or medium education, the probability of separation increases and remains high until age 40 . In contrast, for low educated women it reaches its maximum at age 35 and falls thereafter, corresponding to an increase in the probability of post-separation cohabitation.

Class two broadly represents a long-term cohabitation pattern. Most women at all educational levels form cohabiting relationships from their early 20 s, with a peak in the probability of cohabitation around the age of 28 for high and medium educated women and at age 25 for low educated women. Thereafter, the probability of being in a cohabiting relationship decreases among women with high and medium education coinciding with an increasing probability of marriage from around age 31 (which reaches 0.45 for highly educated women and 0.39 for women with medium education). In contrast, women with low education continue to exhibit a high probability of cohabitation (nearly 0.7 at age 40). Consequently, the corresponding increase in the probability of marriage is limited, reaching only 0.2 by age 40 . This result indicates that low educated women are less likely to formalise their unions. Additionally, the probability of separation is more than twice as high among low educated women as among their more educated counterparts.

Class three describes a generally early transition to marriage with some pre-marital cohabitation. Women with high and medium education have very similar partnership experiences: partnership formation begins with a small bump in the probability of cohabitation, followed by a transition to marriage with a $50 \%$ chance of being married around age 22 . The probability of marriage is close to 1 among these women in their late $20 \mathrm{~s}$ and it remains high with little evidence of separation. The patterns are slightly different for women with low education. Entry into partnership occurs earlier, with a decline in the probability of being single already from age 15 . The probability of pre-marital cohabitation is higher among low educated women than among their more educated counterparts (peaking around 0.3 compared to less than 0.2 for both medium and high educated women).

Class four represents the most 'modern' partnership form with a considerably high incidence of cohabitation before marriage, with a peak at age 25 , when the probability of cohabitation is roughly 0.4. Thereafter, many unions translate into marriage, the probability of which peaks around age 31. We observe roughly similar patterns of partnership formation for women of all educational levels but there are differences in the timing of different partnership transitions. Women with low education tend to enter partnerships later than the more educated. Among low educated women, the probability of remaining never partnered stays close to 1 until age 21 while among medium and high educated women, this happens around age 18 . 
Additionally, women with low education are less likely to experience cohabitation before marriage; the peak of the probability of being in a cohabiting relationship is roughly 0.35 , compared to 0.40 and 0.47 for women with high and medium education, respectively.

Finally, class five captures a more complex pattern of late partnership formation. Regardless of educational level, the probability of being never partnered does not decline until after age 25 and it never falls below 0.2 . After age 25 , union forms are varied; the most and the least educated are more likely to form cohabiting unions than marriages at all ages while women with medium education are more likely to be married after age 37 . Finally, there is some evidence of union instability in this class at later ages.
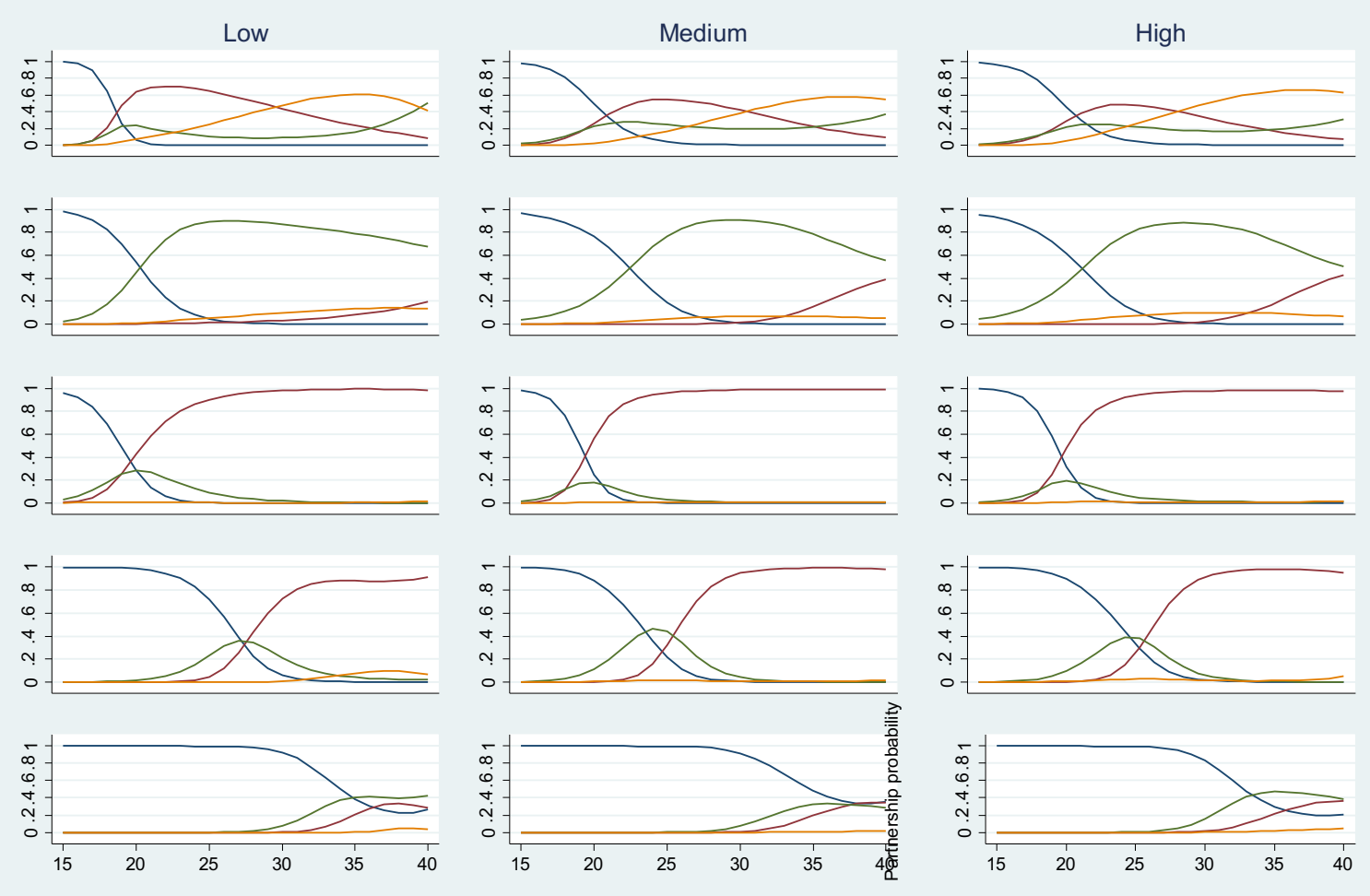

\begin{tabular}{llll}
\hline Never partnered & Marriage & Cohabitation & Separated \\
\hline
\end{tabular}

Note: Each row represents a latent class.

Note: Class 1: Early, varied partnerships (30.7\%); Class 2: Early cohabitation with late transition to marriage (12.6\%); Class 3: Early marriage with some cohabitation (21.5\%); Class 4: Marriage preceded by cohabitation (25.0\%); Class 5: Late and heterogeneous partnership forms (10.0\%).

\section{Figure 3. Results of the five-class Latent Class Growth Models by education (predicted proportion of women in each class)}

To further facilitate the interpretation of educational differences across classes, table 5 presents the predicted probabilities of class membership by education. This table indicates that medium and highly educated women have a higher probability to belong to the 'early marriage with some cohabitation' class (class three) and to the 'marriage preceded by cohabitation' class (class four) than their low educated counterparts. Additionally, low educated women have a much higher probability (0.52) to belong to the 'early, varied partnerships class' (class one) compared to 
those with medium education or higher $(0.15$ and 0.11 , respectively). Last, we did not find large educational differences in the predicted probability of belonging to class two ('early cohabitation with late transition to marriage') and class five ('late and heterogeneous partnerships'). These results indicate that women with higher educational attainment generally experience more complex partnership patterns. The modal class for women with low education is class one ('early, varied partnerships') while for medium and highly educated women it is class three ('early marriage with some cohabitation') although their probability to belong to class four ('marriage preceded by cohabitation') is also larger than that of the other classes.

Table 5. Predicted probability of class membership by educational level

\begin{tabular}{lccc}
\multicolumn{1}{c}{ Class } & \multicolumn{3}{c}{ Educational level } \\
& Low & Medium & High \\
\hline 1 Early, varied partnerships & 0.52 & 0.15 & 0.11 \\
2 Early cohabitation with late transition to marriage & 0.15 & 0.12 & 0.12 \\
3 Early marriage with some cohabitation & 0.11 & 0.34 & 0.39 \\
4 Marriage preceded by cohabitation & 0.13 & 0.28 & 0.29 \\
5 Late and heterogeneous partnership forms & 0.09 & 0.11 & 0.09 \\
\hline
\end{tabular}

\section{Multi-state Event History Model}

Table 6 describes the number of women who were at risk of each transition (total entering) and the number and proportion of those who experienced them. In the examined sample, $70 \%$ of never partnered women formed a cohabiting union while $28 \%$ got married. The remaining $9 \%$ of never partnered women remains never partnered until the end of the observation. Over two thirds of cohabiting unions transitioned to marriage while $22 \%$ ended in union dissolution. A similar proportion (25-26\%) of marriages (both direct marriage and marriage that was preceded by cohabitation) ended with union dissolution. Finally, $75 \%$ of women who experienced union dissolution formed a new partnership.

Table 6. Number (and proportion, \%) of women who experience each partnership transition

Destination state

\begin{tabular}{|c|c|c|c|c|c|c|c|c|c|}
\hline & & $S$ & $C$ & $M$ & $\mathrm{CM}$ & D & $\mathrm{R}$ & $\begin{array}{c}\text { no } \\
\text { event }\end{array}$ & $\begin{array}{c}\text { total } \\
\text { entering }\end{array}$ \\
\hline $\begin{array}{l}0 \\
r\end{array}$ & $S$ & 0 & $\begin{array}{c}908 \\
(70 \%)\end{array}$ & $\begin{array}{c}363 \\
(28 \%)\end{array}$ & 0 & 0 & 0 & $\begin{array}{c}19 \\
(2 \%)\end{array}$ & 1290 \\
\hline $\begin{array}{l}\mathrm{i} \\
\mathrm{g}\end{array}$ & $C$ & 0 & 0 & 0 & $\begin{array}{c}621 \\
(68 \%)\end{array}$ & $\begin{array}{c}202 \\
(22 \%)\end{array}$ & 0 & $\begin{array}{c}85 \\
(9 \%)\end{array}$ & 908 \\
\hline $\mathrm{i}$ & $M$ & 0 & 0 & 0 & 0 & $\begin{array}{c}91 \\
(25 \%)\end{array}$ & 0 & $\begin{array}{c}272 \\
(75 \%)\end{array}$ & 363 \\
\hline $\mathrm{s}$ & $\mathrm{CM}$ & 0 & 0 & 0 & 0 & $\begin{array}{c}163 \\
(26 \%)\end{array}$ & 0 & $\begin{array}{c}458 \\
(74 \%)\end{array}$ & 621 \\
\hline $\begin{array}{l}t \\
a\end{array}$ & $D$ & 0 & 0 & 0 & 0 & 0 & $\begin{array}{c}343 \\
(75 \%)\end{array}$ & $\begin{array}{c}113 \\
(25 \%)\end{array}$ & 456 \\
\hline $\begin{array}{l}\mathrm{t} \\
\mathrm{e}\end{array}$ & $\mathrm{R}$ & 0 & 0 & 0 & 0 & 0 & 0 & 0 & 0 \\
\hline
\end{tabular}


The results of the multi-state event history model are summarised in table 7. Higher educated never partnered women have a higher risk of entering cohabitation and direct marriage than medium and low educated. Furthermore, education has a positive gradient on the transition from cohabitation to marriage; low and medium educated cohabiting women are $45 \%$ and $32 \%$ less likely, respectively, than their highly educated counterparts to marry their cohabiting partner. Following union dissolution, women with low education are less likely to find a new partner compared to highly educated women. Education does not have a significant influence on the dissolution of a cohabiting or a marital union (whether or not it was preceded by cohabitation).

Table 7. Result of the multi-state event history model, hazard ratios

\begin{tabular}{llllllll} 
& $\mathrm{S} \rightarrow \mathrm{C}$ & $\mathrm{S} \rightarrow \mathrm{M}$ & $\mathrm{C} \rightarrow \mathrm{CM}$ & $\mathrm{C} \rightarrow \mathrm{D}$ & $\mathrm{M} \rightarrow \mathrm{D}$ & $\mathrm{CM} \rightarrow \mathrm{D}$ & $\mathrm{D} \rightarrow \mathrm{R}$ \\
\hline Education & & & & & & & \\
Low & $0.73^{*}$ & $0.47^{* * *}$ & $0.55^{* * *}$ & 1.27 & 1.13 & 1.31 & $0.68^{*}$ \\
Medium & $0.70^{* *}$ & $0.59^{* *}$ & $0.68^{* *}$ & 1.23 & 0.98 & 1.29 & 0.88 \\
High (ref) & & & & & & \\
\hline Note: ${ }^{*} p<.05^{* *} p<.01^{* * *} p<.001$ & &
\end{tabular}

\section{Conclusion and Discussion}

This paper qualitatively compared three methodological approaches (i.e. sequence analysis, latent class growth models, and multi-state event history models) to the analysis of life course data focusing on the influence of education on partnership experiences of Norwegian women born between 1955 and 1964. These methods have several similarities and differences. For example, sequence analysis and latent class growth models establish the relationship between education and the probability of belonging to certain groups (clusters or classes) based on women's partnership experiences. In our application, sequence analysis revealed six clusters based on women's partnership experiences (late, varied partnerships; early, direct, and unstable marriage; cohabitation; late, direct, and stable marriage; cohabitation followed by marriage; and early, direct, and stable marriage), latent class growth models suggested five partnership classes (early, varied partnerships; early cohabitation with late transition to marriage; early marriage with some cohabitation; marriage preceded by cohabitation; and late, heterogeneous partnership forms). Multi-state event history models do not classify individuals but rather examine the influence of education on each partnership transition thereby enabling us to draw conclusions about the changing influence of education over the early family life course.
Overall, the examined methods arrive at similar conclusions with respect to the influence of education on partnership experiences. For example, all three methods found that women with higher education are more likely to marry their cohabiting partner. Moreover, sequence analysis showed that the lower educated are more likely to belong to early union formation clusters than women with high education, who are more likely to form direct, stable marriages at later ages. This is in line with findings of LCGMs, which showed that low educated women are the most likely to belong to the 'early, varied partnerships' class (class one). Additionally, the results of multi-state models revealed that the more educated have a higher risk of experiencing direct marriage than the lower educated. Multi-state models showed that more educated women have a higher risk of finding a new partner following union dissolution than their lower educated counterparts. However, the results of the LCGMs did not suggest significant educational differences in the probability of belonging to the 'late and heterogeneous partnerships' class (class five). Similarly, we found no significant educational differences between the likelihood of belonging to the 'late, varied partnerships' cluster (cluster one) and the 'early, direct, and stable marriage' cluster (cluster six). Additionally, multi-state event history models showed that never partnered highly educated women have higher risks to enter 
cohabitation than their lower educated counterparts. At the same time, these women are also more likely to marry their cohabiting partner and thus less likely to remain cohabiting. However, LCGMs did not find educational differences in the probability of belonging to class two ('early cohabitation with late transition to marriage').

The examined methods have different properties and approach studying the life course in a different way. In order to emphasise the strengths of each technique and to accommodate their limitations, the presented analyses could not have been implemented in exactly the same way for the three techniques. For example, the multi-state event history model and sequence analysis were estimated using monthly data while the LCGMs relied on yearly data due to computational issues. This implies that in LCGMs the number of transitions might be underestimated and some variation in life courses might be lost. Additionally, the multi-state event history model incorporated a time-varying education variable while the other two methods investigated the association between the highest level of education at the time of the interview (i.e. a time-constant variable) and partnership formation. Finally, while the multi-state event history model estimated the influence of education on first and higher order partnership transitions separately, the order of union is encoded in the sequences for LCGMs and SA.

These differences in the implementation of the analyses could potentially explain some of the differences in the results of the multi-state event history model and the other two methods but they cannot account for differences between the results of SA and LCGMs. However, it has been shown that SA and LCGMs should not be expected to give the same answer. Using simulated data, Warren, Luo, Halpern-Manners, Raymo, and Palloni (2015) showed that the number of trajectories these methods produce might differ from each other as well as from the true number of trajectories. Moreover, LCGMs and SA might assign the same individuals to different trajectory groups.

Another possible explanation for the differences in the results produced by the three methods may be related to specific choices made by the researcher during different stages of the analyses. For example, in sequence analysis, the researcher has an array of options to calculate the distance matrix, to define insertion, deletion, and substitution costs, as well as to perform the clustering. Each of these decisions might influence the outcome of sequence analysis. Similarly, in case of LCGMs, the method chosen to create the classes may alter the resulting latent classes. In case of the multi-state event history analysis, there is no need to make such arbitrary choices.

Even though the applications are not exactly the same and occasionally they provide somewhat different results, by illustrating the properties and application of the different techniques, we were able to identify similarities and differences between these methods with respect to their ability to address certain desirable aspects of studying the family life course. These are summarised in table 8. 
Table 8. Summary of the properties of sequence analysis, latent class growth models, and multistate event history analysis

\begin{tabular}{|c|c|c|c|}
\hline & SA & LCGM & $\begin{array}{c}\text { Multi-state Event } \\
\text { History model }\end{array}$ \\
\hline Transition intensities & $(\sqrt{ })$ & $x$ & $\checkmark$ \\
\hline Classifying individuals & $\checkmark$ & $\checkmark$ & $x$ \\
\hline Covariate information alters pattern & $x$ & $\checkmark$ & $\checkmark$ \\
\hline Computationally simple & $\checkmark$ & $x$ & $\checkmark$ \\
\hline Time-varying covariates & $x$ & $x$ & $\checkmark$ \\
\hline Model based & $x$ & $\checkmark$ & $\checkmark$ \\
\hline $\begin{array}{l}\text { Protection against baseline } \\
\text { misspecification }\end{array}$ & $\checkmark$ & $x$ & $\checkmark$ \\
\hline Possibility to incorporate weights & $(\checkmark)$ & $\checkmark$ & $x$ \\
\hline
\end{tabular}

Note: The given method is $\checkmark$ able to, $\boldsymbol{x}$ not able to or $(\checkmark)$ partially able to deal with this dimension of the family life course.

Based on this table, we can formulate broad recommendations for researchers choosing between different life course methods. First, sequence analysis is best applied to research questions which attempt to describe partnership behaviours of different groups of women and the overall associations of these groups with certain covariates. This can be achieved through the method's ability to classify individuals and allow for covariates to predict women's membership in the different clusters. Overall, fitting the model does not require a lot of computing power and because the procedure is not model based, the user is protected against baseline misspecification (i.e. no baseline needs to be specified). Although not presented in this paper, the method can also calculate transition intensities between the different states. As it is not possible to condition sequences, or more importantly transition probabilities, on covariate information or to allow for the incorporation of changing covariate information over the life course, this method cannot answer research questions relating to the changing influence of a variable over the life course. In other words, whereas LCGMs and multi-state event history models directly involve covariates thereby providing a better assessment of the net effect of a covariate of interest on different family life transitions, SA is not able to directly incorporate covariates. Last, it is not possible to take into account survey weights while computing distances between sequences (by definition this is a one to one comparison) although weights can be incorporated when comparing cluster sizes and in regression models.

Second, latent class growth models have a number of similar properties to sequence analysis. Its main advantage is that it is able to incorporate more complicated structures by, for example, allowing for covariate information to alter the shape of partnership trajectories. Additionally, LCGM is the only one among the three examined methods which allows the researcher to fully incorporate survey weights in the analysis. Unfortunately, the implementation of LCGMs is computationally intense and requires considerable computing power to estimate models for large datasets. Moreover, as LCGMs are model based, a greater degree of robustness is required particularly when estimating the shape of the growth curves. 
On the other hand, this also means that a greater variety of fit-statistics is available than in sequence analysis, where the decision of the optimal number of clusters is more arbitrary than in LCGMs. Last, it should be noted that while LCGMs allow for testing the model performance via simulation approaches (e.g. Nylund et al., 2007), such a test is not available for sequence analysis. Thus, LCGMs are most suited to studying complex research topics where the aim is to identify differences in covariate effects between groups of individuals. The present paper has demonstrated this by extracting different classes of partnership behaviour and comparing the effect of educational attainment within these classes.

Finally, although multi-state event history models do not classify individuals in the same way as the previous two techniques, there are a number of distinct advantages to using this method. For example, the estimation of transition intensities allows for examining several transitions over the life course within the same model as well as for estimating the changing influence of covariates over the life course by allowing for the incorporation of time-varying covariates. This is one of the key advantages of multi-state event history models as neither sequence analysis, nor latent class growth models are capable of studying changing covariate effects over the life course. Additionally, the use of a stratified Cox model provides some protection against baseline misspecification. However, currently, it is not possible to incorporate survey weights using the mstate package. To conclude, multi-state event history models can best answer research questions specifically related to changing covariate effects over the life course. For example, as this paper has shown, it can estimate the changing influence of education on different partnership transitions over the early family life course.

The analyses presented in this study have some limitations. First, the multi-state event history model assumes that the hazards of the examined transitions for women with different educational level are proportional. This assumption might not be realistic. The multi-state event history model would allow for the incorporation of interaction effects between age and education in order to relax the assumption of proportional hazards. However, LCGMs and SA are unable to explicitly incorporate such interactions. ${ }^{\text {viii }}$ To keep the models comparable, we refrained from including interactions between age and education in the multi-state event history model. Second, next to education, many factors may influence the timing and sequencing of partnership transitions. For LCGMs and SA, which included a time-constant education variable, the influence of other time-constant covariates on the timing and sequencing of the examined transitions could have been studied. However, in the examined dataset time-varying information, which was used in the multi-state event history model, could only be reconstructed for education. Including more covariates for LCGMs and SA but not for the multistate event history model would not have facilitated the comparison of the methods and the results they produce. Researchers can build on this simple application and perform more complex analyses. Last, rather than comparing the three methods based on goodness of fit statistics or applying them to the same simulated dataset, we took a more applied approach and qualitatively compared the advantages and disadvantages of the three techniques. This approach was in line with the aim of the paper, namely, to give a qualitative overview of the strengths and weaknesses of these techniques and their applicability to answering different research questions.

Taken together, by comparing sequence analysis, latent class growth models, and multi-state event history models, this paper contributed to the discussion on the applicability of different methods for studying the life course. We showed that latent class growth models and multi-state event history models are a useful addition to life course researchers' methodological toolkit and that these methods can address certain research questions better than the more commonly applied sequence analysis or simple event history analysis. In particular, we have stressed the types of research questions that may be better addressed using these techniques which provide new insights in the field of life course studies. 


\section{Acknowledgements}

Júlia Mikolai was a PhD student at the Department of Social Statistics and Demography at the University of Southampton and was funded by $a+3$ Scholarship provided by the Economic and Social Research Council (ES/J500161/1) while completing most of this work. Mark Lyons-Amos was a post-doctoral research fellow at the University of Southampton, funded by the European Research Council Starting Grant CHILDCOHAB. The authors would like to thank Dr Agnese Vitali for her comments and suggestions on a previous draft of this paper. The Harmonized Histories data file was created by the Non-Marital Childbearing Network (http://www.nonmarital.org). The GGS data were obtained from the GGP Data Archive and refer to the publication that contains the model survey instruments: United Nations 2005. Generations \& Gender Programme: Survey Instruments. New York and Geneva: UN, 2005. The authors acknowledge the use of the IRIDIS High Performance Computing Facility and associated support services at the University of Southampton in the completion of this work.

\section{References}

Abbott, A., \& Tsay, A. (2000). Sequence analysis and optimal matching methods in sociology: Review and prospect. Sociological Methods \& Research, 29(1), 3-33. https://doi.org/10.1177/0049124100029001001

Andersen, P. K., \& Keiding, N. (2002). Multi-state models for event history analysis. Statistical Methods in Medical Research, 11(2), 91-115. https://doi.org/10.1191/0962280202SM276ra

Baizán, P., Aassve, A., \& Billari, F. C. (2003). Cohabitation, marriage, and first birth: The interrelationship of family formation events in Spain. European Journal of Population, 19(2), 147-169. https://doi.org/10.1023/A:1023343001627

Baizán, P., Aassve, A., \& Billari, F. C. (2004). The interrelations between cohabitation, marriage and first birth in Germany and Sweden. Population and Environment, 25(6), 531-560. https://doi.org/10.1023/B:POEN.0000039064.65655.3b

Barban, N., \& Billari, F. C. (2012). Classifying life course trajectories: A comparison of latent class and sequence analysis. Journal of the Royal Statistical Society Series C Applied Statistics, 61, 765-784. https://doi.org/10.1111/j.1467-9876.2012.01047.x

Billari, F. C. (2001a). The analysis of early life courses: Complex descriptions of the transition to adulthood. Journal of Population Research, 18(2), 119-142. https://doi.org/10.1007/BF03031885

Billari, F. C. (2001b). Sequence analysis in demographic research. Canadian Studies in Population, 28(2), 439458.

Billari, F. C. (2005). Life course analysis: Two (complementary) cultures? Some reflections with examples from the analysis of the transition to adulthood. Advances in Life Course Research, 10, 261-281. https://doi.org/10.1016/S1040-2608(05)10010-0

Billari, F. C., \& Piccarreta, R. (2001). Life courses as sequences: an experiment in classification via monothetic divisive algorithms. In S. Borra, R. Rocchi, M. Vichi \& M. Schader (Eds.), Advances in Classification and Data Analysis (pp. 351-358). Berlin and New York: Springer Verlag. https://doi.org/10.1007/978-3642-59471-7_43

Billari, F. C., \& Piccarreta, R. (2005). Analyzing demographic life courses through sequence analysis. Mathematical Population Studies, 12(2), 81-106. https://doi.org/10.1080/08898480590932287

Bonetti, M., Piccarreta, R., \& Salford, G. (2013). Parametric and nonparametric analysis of life courses: An application to family formation patterns. Demography, 50(3), 881-902. https://doi.org/10.1007/s13524-012-0191-z

Brzinsky-Fay, C., \& Kohler, U. (2010). New developments in sequence analysis. Sociological Methods \& Research, 38(3), 359-364. https://doi.org/10.1177/0049124110363371

Brzinsky-Fay, C., Kohler, U., \& Luniak, M. (2006). Sequence analysis with Stata. The Stata Journal, 6(4), 435460.

Calinski, R. B., \& Harabasz, J. A. (1974). A dendrite method for cluster analysis. Communications in StatisticsTheory and Methods, 3(1), 1-27. https://doi.org/10.1080/03610927408827101 
de Wreede, L. C., Fiocco, M., \& Putter, H. (2011). mstate: An R package for the analysis of competing risks and multi-state models. Journal of Statistical Software, 38(7), 1-30.

https://doi.org/10.18637/jss.v038.i07

Duda, R. O., \& Hart, P. E. (1973). Pattern Classification and Scene Analysis. New York: Wiley.

Elzinga, C. H., \& Liefbroer, A. C. (2007). De-standardization of family-life trajectories of young adults: A crossnational comparison using sequence analysis. European Journal of Population, 23(3-4), 225-250. https://doi.org/10.1007/s10680-007-9133-7

Halpin, B. (2014). SADI: Sequence analysis tools for Stata, Working Paper WP2014-03, Department of Sociology, University of Limerick, http://www.ul.ie/sociology/pubs/wp2014-03.pdf.

Heuveline, P., \& Timberlake, J. M. (2004). The role of cohabitation in family formation: The United States in comparative perspective. Journal of Marriage and Family, 66, 1214-1230.

https://doi.org/10.1111/j.0022-2445.2004.00088.x

Hougaard, P. (1999). Multi-state models: A review. Lifetime Data Analysis, 5(3), 239-264. https://doi.org/10.1023/A:1009672031531

Jung, T., \& Wickrama, K. A. S. (2008). An introduction to Latent Class Growth Analysis and Growth Mixture Modeling. Social and Personality Psychology Compass, 2(1), 302-317. https://doi.org/10.1111/j.1751-9004.2007.00054.x

Nylund, K. L., Asparouhav, T., \& Muthen, B. O. (2007). Deciding on the number of classes in Latent Class Analysis and Growth Mixture Modeling: A Monte Carlo simulation study. Structural Equation Modeling: A Multidisciplinary Journal, 14(4), 535-569. https://doi.org/10.1080/10705510701575396

Perelli-Harris, B., \& Gerber, T. P. (2011). Nonmarital childbearing in Russia: Second Demographic Transition or Pattern of Disadvantage? Demography, 48(1), 317-342. https://doi.org/10.1007/s13524-0100001-4

Perelli-Harris, B., Kreyenfeld, M., \& Kubisch, K. (2010). Harmonized Histories: Manual for the preparation of comparative fertility and union histories. Rostock, MPIDR Working Paper WP-2010-011.

Perelli-Harris, B., \& Lyons-Amos, M. (2015). Changes in partnership patterns across the life course: An examination of 14 countries in Europe and the United States. Demographic Research, 33, 145-178. https://doi.org/10.4054/DemRes.2015.33.6

Perelli-Harris, B., Sigle-Rushton, W., Kreyenfeld, M., Lappegård, T., Keizer, R., \& Berghammer, C. (2010). The educational gradient of childbearing within cohabitation in Europe. Population and Development Review, 36(4), 775-801. https://doi.org/10.1111/j.1728-4457.2010.00357.x

Piccarreta, R., \& Billari, F. C. (2007). Clustering work and family trajectories using a divisive algorithm. Journal of Royal Statistical Society, A, 170(4), 1061-1078. https://doi.org/10.1111/j.1467-985X.2007.00495.X

Putter, H., Fiocco, M., \& Geskus, R. B. (2007). Tutorial in biostatistics: Competing risks and multi-state models. Statistics in Medicine, 26(11), 2389-2430. https://doi.org/10.1002/sim.2712

Putter, H., van der Hage, J., de Bock, G. H., Elgalta, R., \& van de Velde, C. J. H. (2006). Estimation and prediction in a multi-state model for breast cancer. Biometrical Journal, 48(3), 366-380. https://doi.org/10.1002/bimj.200510218

Steele, F., Kallis, C., Goldstein, H., \& Joshi, H. (2005). The relationship between childbearing and transitions from marriage and cohabitation in Britain. Demography, 42(4), 647-673. https://doi.org/10.1353/dem.2005.0038

Wang, C. P., Hendricks Brown, C., \& Bandeen-Roche, K. (2005). Residual diagnostics for growth mixture models: examining the impact of a preventive intervention on multiple trajectories of aggressive behavior. Journal of the American Statistical Association, 100(471), 1054-1076. https://doi.org/10.1198/016214505000000501

Warren, J. R., Luo, L., Halpern-Manners, A., Raymo, J., \& Palloni, A. (2015). Do different methods for modelling age-graded trajectories yield consistent and valid results? American Journal of Sociology, 120(6), 1809-1856. https://doi.org/10.1086/681962 


\section{Endnotes}

'This paper used the version that is available in the Harmonized Histories (Perelli-Harris, Kreyenfeld, \& Kubisch, 2010).

ii Throughout this study never partnered women are defined as those who have never lived in a coresidential union for at least three months.

iii $\frac{1}{\left(\frac{e(2)}{J e(1)}\right)}=1+\frac{T^{2}}{N_{1}+N_{2}-2}$, where $N_{C}$ denotes the number of observations in cluster $c$.

iv If two partnership states are present in the same year, the higher value is selected. This means that short episodes of e.g. cohabitation or being separated before re-partnering will be missed.

${ }^{v}$ We use yearly (instead of monthly) intervals to reduce the size of the dataset and to increase the speed of estimation. Robustness checks for similar analyses have shown that the reduction of information from monthly to yearly intervals do not substantially influence the results (Perelli-Harris \& Lyons-Amos, 2015).

${ }^{v i}$ Exploratory analyses revealed that higher order classes tend to be sparsely populated with limited interpretability.

vii Although it can be argued that multi-state models predict group membership in terms of state occupation probabilities, in multi-state models individuals move from one state to the next. However, in SA and LCGMs each individual can only belong to one cluster or class.

viii It would be possible to build sequences of changes in educational level and examine these sequences together with sequences of partnership states. 